\title{
Applications of Learning Methods to imaging issues in Archaeology, regarding ancient ceramic manufacturing
}

\author{
K. Dia*1,3， V.L. Coli ${ }^{1,3}$ ， L. Blanc-Féraud, J. Leblond, ${ }^{3}$ L. Gomart, ${ }^{4}$ D. Binder ${ }^{1}$
}

July 27, 2021

Keywords: Machine Learning algorithms, Convolution Neural Network, Support Vector Machine, Image classification and analysis, Hough transform, Archaeology, Neolithic Pottery.

\begin{abstract}
Archaeological studies involve more and more numerical data analyses. In this work, we are interested in the analysis and classification of ceramic sherds tomographic images in order to help archaeologists in learning about the fabrication processes of ancient pottery. More specifically, a particular manufacturing process (spiral patchwork) has recently been discovered in early Neolithic Mediterranean sites, along with a more traditional coiling technique. It has been shown that the ceramic pore distribution in available tomographic images of both archaeological and experimental samples can reveal which manufacturing technique was used. Indeed, with the spiral patchwork, the pores exhibit spiral-like behaviours, whereas with the traditional one, they are distributed along parallel lines, especially in the experimental samples. However, in archaeological samples, these distributions are very noisy, making analysis and discrimination hard to process. Here, we investigate how Learning Methods (Deep Learning and Support Vector Machine) can be used to answer these numerically difficult problems. In particular, we study how the results depend on the input data (either raw data at the output of the tomographic device, or after a preliminary pore segmentation step), and the quality of the information they could provide to archaeologists.
\end{abstract}

\section{Introduction}

Back in the seventh and sixth millennia BC, when farming was introduced to Europe, pottery was a central practice for storing and food processing. For the archaeologists, the techniques used to manufacture ancient pottery can act as a powerful marker of the social identity of their producers. A previously unrecognised pottery manufacturing technique was recently identified (Gomart et al., 2017) at the Pendimoun rock shelter (early Neolithic layers dated to the early 6th millennium

\footnotetext{
${ }^{*}$ Corresponding author

${ }^{1}$ University Côte d'Azur, CNRS, CEPAM, 06300 Nice, France

${ }^{2}$ University Côte d'Azur, CNRS, INRIA, I3S Lab., Sophia Antipolis, France

${ }^{3}$ University Côte d'Azur, INRIA, Team Factas, B.P. 93, 06902 Sophia Antipolis Cedex, France

${ }^{4}$ University Paris 1 Panthéon-Sorbonne, CNRS-UMR 8215 Trajectoires, 92023 Nanterre, France
} 

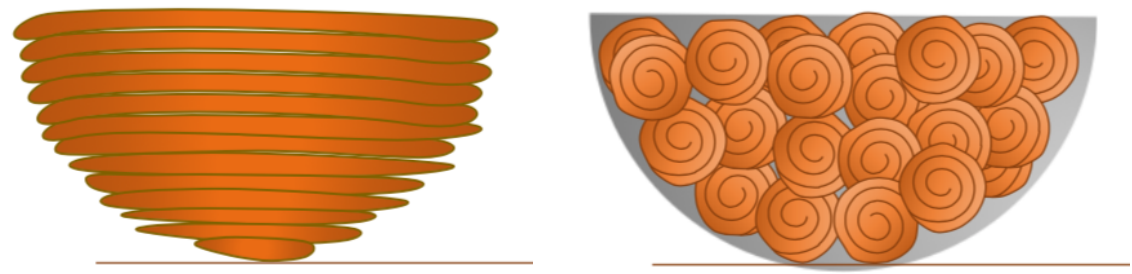

Figure 1: Coiling Technology (superimposition of coils, left), Spiral Patchwork Technology (juxtaposed spiral patches, right).

BCE) in southeastern France (Alpes-Maritimes) and named spiralled patchwork technology (SPT). The construction of pottery using SPT relies on juxtaposing and fusing circular patches, see Figure 1, right. This manufacturing technique is contemporary to the coiling technology (CoT), which is often observed in European early farming contexts (Salanova et al., 2010; Gomart, 2014; Gomart, 2010) and is characterised by superimposition of coils, see Figure 1, left.

Micro-computed tomographic ( $\mu$-CT) 3-D images of pottery sherds formed using these techniques were acquired, revealing their internal structure. In particular, in 2-D cross-sections, the distribution of pores (i.e., voids trapped inside the clay forming the sherd structure during the building process) revealed curvilinear patterns for the SPT sherds and mainly parallel linear patterns for the CoT sherds, see Figure 2 (mostly visible in the first and second columns).

The goal of the present work is to analyse and classify pottery manufacturing sequences using the images acquired by $\mu$-CT from available archaeological sherds and from experimental sherds that were recently manufactured in controlled conditions using both coiling and spiral patchwork techniques. These scans allow to access the complete internal structure of these sherds and, after a segmentation process, to isolate the regions corresponding to pores, showing their spatial distribution, see Figure 2, bottom line.

The purpose of this work is to provide a quantitative characterisation of $\mathrm{Ne}$ olithic pottery forming techniques using 3-D datasets. To the authors knowledge, it represents a first attempt to achieve this goal.

The dataset considered in this study is composed of sixteen sherds:

- four CoT sherds (one archaeological and three experimental sherds);

- twelve SPT sherds (five archaeological and seven experimental sherds).

The first and second columns of Figure 2, which show 2-D cross-sections images of CoT and SPT experimental sherds, reveal that pore distribution varies according to the manufacturing technique used. The third and fourth columns of Figure 2, which represent archaeological CoT and SPT sherds, show that manufacturing technique is much more difficult to detect in archaeological samples as patterns are not visually distinguishable, most probably because of fragment alteration through time. The pore distribution of both archaeological and experimental sherds is accessible by analysing the segmented images (as in Figure 2, bottom line). Detecting pore alignments, which are visible in Figure 2 down-left image, allows to identify the CoT technique. This is obtained by using the Hough transform as in (Coli et al., 2021), an automatic way to detect aligned patterns in images. We first select the lines that intersect the highest quantity of pore regions, and we analyse their parallelism by computing the scalar product between their directional vectors. As expected, CoT-like images reveal parallel lines characterised by values of the scalar products close to 1 , whereas other techniques as SPT show other / random 
distributions, see (Dia, 2020).
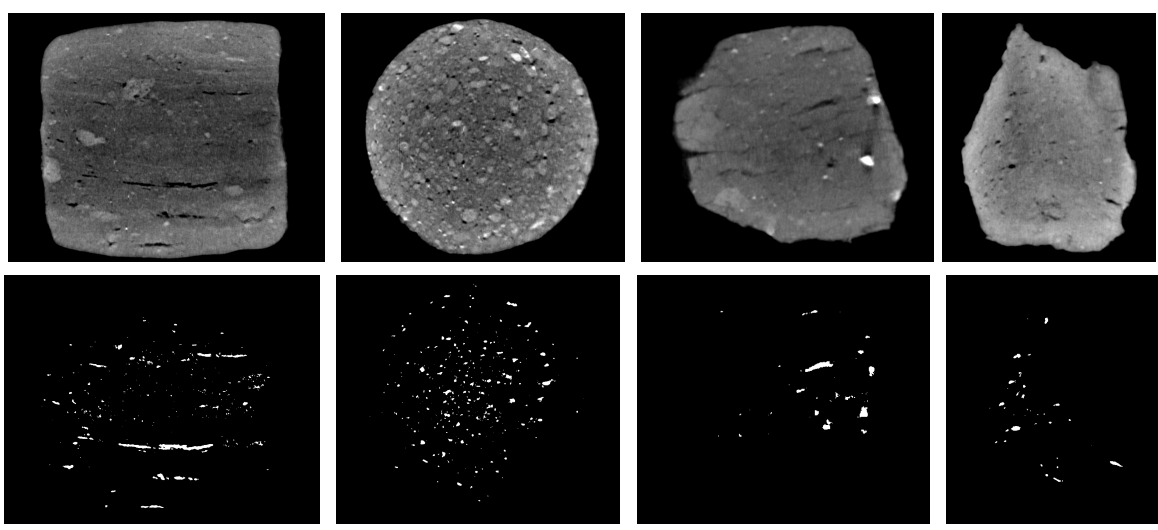

Figure 2: Pores segmentation results. The first line shows 2-D tomographic images and the second line the corresponding segmented images. From left to right: CoT and SPT experimental sherds, CoT and SPT archaeological sherds.

To classify ceramic sherd images, we investigate in this work classical Machine Learning (ML) algorithms. The Support Vector Machine (SVM) method (Widodo and Yang, 2007) is applied to preliminary features given by the scalar products between the detected lines, while Deep Learning (DL) method is initially run on the segmented images (extracted pores). We use Convolutional Neural Network (CNN) (Krizhevsky et al., 2012) with the pre-trained network GoogLeNet (Szegedy et al., 2015) for transfer learning to classify segmented images. Finally, as CNN-based methods result in automatic feature extraction, the pore segmentation step appears not be necessary. The same CNN based classification procedure, with the learning phase, has been applied directly on the 2D slices of tomographic images and good classification results have been obtained.

Below, we describe the data, the considered analytical protocol and classification methods, and show how they can answer archaeological questions. In Section 2 we precise some information on data acquisition and treatments. Section 3 is devoted to a brief overview of the exploited learning algorithms (ML/SVM and DL/CNN). In Section 4, classification results for the above dataset including both pottery forming techniques are given and analysed. Finally, a brief conclusion is provided in Section 5.

\section{Data processing}

We describe here the data acquisition process, the pre-processing step of the images, that consists in pore segmentation, and the way we proceed to feature extraction, using Hough transform.

\subsection{Image acquisition}

The experimental and archaeological pottery sherds were scanned using a SkyScan1178 X-ray $\mu$-CT system (Bruker) with two $1280 x 1024$ X-ray cameras, beam energy of $60 \mathrm{kV}$, a $0.5 \mathrm{~mm}$ thick aluminium filter, $0.7^{\circ}$ rotation. Each reconstructed image has a resolution of $81 \mu \mathrm{m}$. To reduce the data size, a box volume surrounding each sherd is cropped from the 3-D stack and used in the analysis. In this work we 
considered central 2-D cross sections of tomographic images of sherds (see Figure 2, first row, for an example of CoT and SPT experimental and archaeological sherds).

\subsection{Image processing}

The spatial distribution of pores inside pottery depends on the manufacturing sequence used, as air tends to be trapped between building elements during the forming process. Thus, CoT sherds are characterised by pores showing linear patterns, while SPT sherds reveal more dispersed patterns, which in some cases suggest circular dispositions by visual interpretation. Therefore, the segmentation of pores inside tomographic images reveals useful information for the characterisation of the building technique.

The procedure we use to segment pore regions is as follows. First, the pixels with low intensity grey-scale values are selected as corresponding to air. Then, a volume binarisation is applied to separate air pixels from the rest of the materials characterising the pottery (i.e. clay and mineral inclusions). Finally, a connected components detection is performed in order to remove unwanted connected components corresponding to the air surrounding the sherd. This procedure is applied to 3-D stack of tomographic images and more details can be found in (Coli et al., 2021). The 2-D cross-section images which we chose to use in this work are successively extracted from the 3-D stack. In Figure 2, bottom line, we show examples of results of this segmentation process, where only the porosity contrast is kept visible in the images. In the rest of this work, we refer to these images as segmented images. After segmentation, the images show mostly aligned patterns for the pores in the CoT sherds, while the distribution appears to be more circular and random for the SPT ones (Figure 2, bottom line). Then, the distribution of pores is investigated by means of the Hough transform.

\subsection{Feature extraction}

The Hough transform is a classical technique which is often used to detect alignments or other patterns in images (Ballard, 1981; Beltrametti et al., 2013; Mukhopadhyay and Chaudhuri, 2015). In order to detect lines in 2-D images, the Hough transform relates points in the image to sinusoidal curves in an associated Hough space. The intersections between sinusoids account for the presence of points along lines in the original image. Keeping the points where most sinusoids intersect provides us with the main alignment directions of pores. In the analysis of the segmented images, only the five lines which have the greater amount of aligned pore pixels are retained in each image for both classes (e.g., see Figure 3). The parallelism between these five lines is tested by computing the scalar products between their directional vectors (Dia, 2020; Coli et al., 2021). These scalar products constitute the main feature which will be provided to the SVM classifier.

A second feature is provided by the number of intersection points between the recovered lines in the image. Indeed, these lines are almost parallel for CoT sherds and may intersect outside the image region, while this is not the case for SPT sherds for which these lines admit quite a few intersection points close to the image centre (see Figure 3).

\section{Classification with learning algorithms}

In this section, we briefly introduce the two classical ML methods we used to classify the dataset. The Support Vector Machine (SVM) (Widodo and Yang, 2007) 

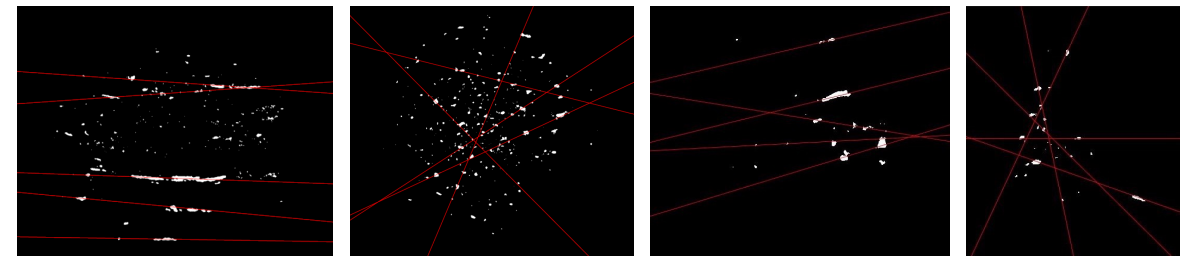

Figure 3: Hough transform results. The red lines represent the detected alignments. From left to right: CoT and SPT experimental sherds, CoT and SPT archaeological sherds .

and Convolutional Neural Network (CNN) (Krizhevsky et al., 2012; de Rezende et al., 2018), both are supervised learning methods, meaning that they require a labeled dataset to be trained. In this study, labels correspond to CoT and SPT techniques. The big difference between these two methods is that a feature extraction procedure is essential for the SVM in order to classify the data. These features may also provide important additional information in view of archaeological interpretations. In this archaeological sherd study, features are based on the alignments in the pores structure. While, $\mathrm{CNN}$ do not require this preliminary process, instead the algorithm detects and extracts necessary features throughout its hidden layers. In the following two sections we provide a short overview of these standard methods in the context of this sherd study.

\subsection{Support Vector Machine}

Support Vector Machine is a ML method used to perform classification tasks. It was initially introduced as a two-class classification technique, which can be extended to multiple-class classifier (Widodo and Yang, 2007; Abe, 2010). For data in a $n$-dimensional space with two classes, the objective of the SVM classifier is to find a hyperplane in the $n$-dimensional space that separates the two classes. Among all possible hyperplanes, the objective is to find the one that has the maximum margin, i.e. the maximum distance to data points of both classes (see Figure 4, left).

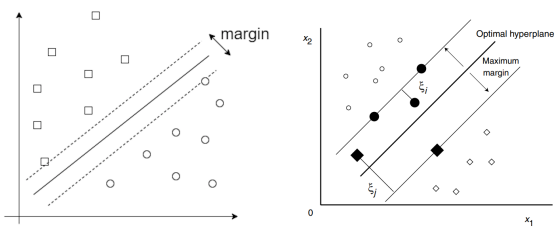

Figure 4: Left: an example of how a dataset is separated in two different classes by a hyperplane. Right: a non-separable case, with an example of slack variables.

Usually, real-life problems contain noisy data and may not be efficiently separated by such a hyperplane. Indeed, if it exists, this hyperplane may have a very small margin (minimal distance to the points, see Figure 4, left). To deal with this problem, an additional term weighted by a parameter $C \in \mathbb{R}^{+}$is introduced in the SVM, in order to specify how many errors can be accepted in the classification. The parameter $C$ allows to tune the trade-off between having a wide margin and the number of admitted errors (that correspond to the slack variables in Figure 4, right).

Moreover, data may not be separable by a hyperplane (see an example Figure 5). In such general cases, they are projected onto a higher dimensional feature 
space, where linear classification is possible. This is done by the use of a kernel transformation such as linear, polynomial and Gaussian Radial Basis Function (RBF) (Widodo and Yang, 2007).

Based on the archaeological question of identifying the CoT or SPT manufacturing techniques on the basis of sherds, it seems natural to first detect alignment of pores in segmented images and then classify data from these lines. More precisely, the considered features for SVM classification are line intersections points and dot products between the Hough-detected lines, as described in Section 2.3. Due to non-linearity of the data, a RBF Kernel, depending on a parameter $\gamma$, is used to increase the accuracy rate. See Section 4 for technical details and values.
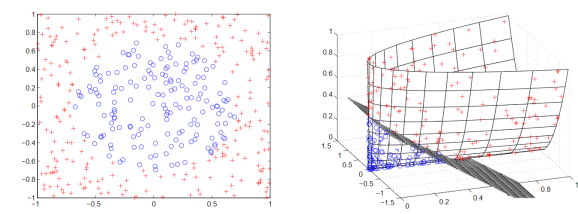

Figure 5: The left picture shows a 2-D set of points impossible to separate using linear SVM. The right picture shows a kernel transformation of these data in a 3-D space where they can be easily separated (https://commons.wikimedia.org/wiki/File:Nonlinear_ SVM_example_illustration.svg).

\subsection{Convolutional Neural Network}

Classification Convolutional Neural Network is a Deep Learning method (Krizhevsky et al., 2012; de Rezende et al., 2018) which generally consists of three main parts (see Figure 6 as an example): an input layer part, where the image is given to the classifier, a feature extraction (or a hidden layer) part, and a classification part that uses fully connected layers (i.e. each neuron of a layer is connected to every neurons of the following layer). This overall network provides as an output the probability values for an input image to belong to each of the classes. In this work two classes are considered, CoT and SPT. CNN have shown unequalled perfor-

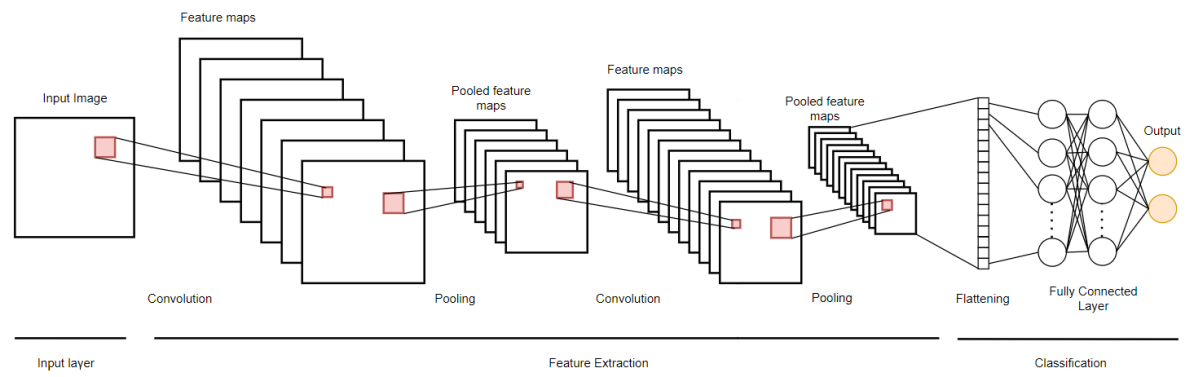

Figure 6: CNN architecture.

mances in image classification. Such performances are mainly due to the learning of the hidden network from a huge number of images. Indeed, a feature extraction network is classically composed of a sequence of layers, each one containing bias addition, convolution filters, activation function (Rectified Linear Unit, ReLu), and pooling operation.

We choose to use a transfer-learning procedure with a pre-modeled and pretrained network, specifically the GoogLeNet architecture (Szegedy et al., 2015). 
The architecture and huge quantity of parameters of the first two parts of this CNN network which compute image characteristics (e.g. bias, coefficients of the convolution filters) remained unchanged. Only the parameters of the third fully connected layer part were estimated and tested using our dataset, which is one of the main advantages of using such a network. For this estimation, we used the DL Matlab toolbox ${ }^{1}$.

All parameters of SVM and CNN are provided in Section 4.

\section{Classification results}
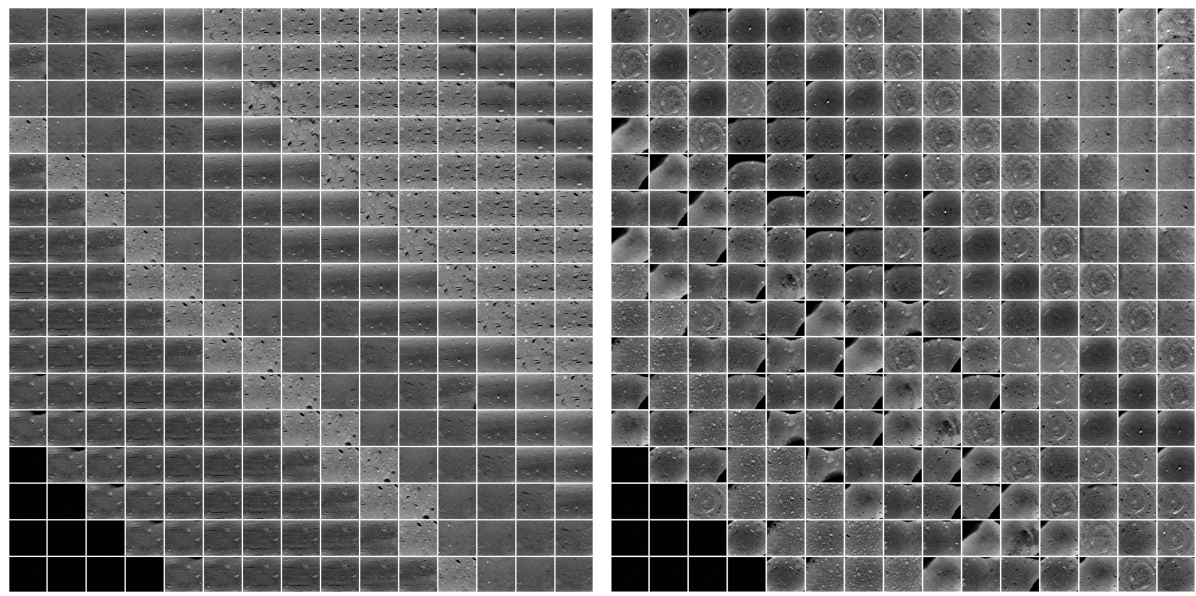

Figure 7: Grey-scale tomographic images, cropped central regions. Left: the $230 \mathrm{im}$ ages composing the CoT dataset. Right: the 230 images composing the SPT dataset. The 10 black images displayed in the bottom-left corners of each set are not included in the datasets.

This section presents the classification results obtained on lines, segmented images (see Section 2.2), and 2-D cross-sections of raw tomographic images. The considered datasets are as follows:

- 360 segmented binary images (130 from CoT sherds and 130 from SPT sherds), used for SVM and CNN classification.

- 460 grey-scale images (230 from CoT sherds and 230 from SPT sherds), used for CNN classification.

Note that the dataset of segmented images is smaller than the dataset of tomographic images, because the pore segmentation process can produce empty images (dark images, which correspond to a slice where no pores were detected).

As Figure 2 (first line) shows, the CoT experimental sherds usually have a squared shape, while the SPT ones are round-shaped. Since this shape is not relevant for manufacturing characterisation, it may induce bias in the classification. To avoid this phenomenon, only the central part of each grey-scale image is considered in the experiments (see Figure 7).

Whatever the classification methods and the input data, the learning step is performed on $80 \%$ of the dataset and the remaining $20 \%$ are used for testing.

\footnotetext{
${ }^{1}$ https://it.mathworks.com/help/deeplearning/ref/trainnetwork.html
} 
Table 1: Prediction accuracy for each object category and all considered classification methods. The number of images in the testing dataset is indicated between parentheses. The results are averaged over 1000 runs for the SVM method.

\begin{tabular}{cccc}
\hline Object category & SVM (segmented images + features) & CNN (segmented images) & CNN (grey-scale images) \\
SPT & $77.7 \%(36)$ & $88.8 \%(36)$ & $100 \%(46)$ \\
CoT & $79.5 \%(36)$ & $100 \%(36)$ & $93.4 \%(46)$ \\
\hline Overall accuracy & $78.6 \%(72)$ & $94.4 \%(72)$ & $96.7 \%(92)$ \\
\hline
\end{tabular}

\subsection{Results with Support Vector Machine}

As mentioned in Section 3.1, a cost parameter $C$ and the Kernel parameter $\gamma$ must be tuned in order to prevent under- or over-fitting phenomena. A grid search is performed to determine the optimal values of these parameters. From these multiple tests, $C$ was set to 100 and $\gamma$ to 10 . The dataset is randomly shuffled 1000 times and different learning and testing sets are picked for each run. The prediction accuracy results averaged over 1000 runs on 72 test images are shown in Table 1.

\subsection{Results with Convolutional Neural Network}

The second method we consider is the classification by CNN, first on segmented images, and then on raw images.

As presented in Section 3.2, we use a transfer-learning procedure with GoogLeNet network which requires three-dimensional $224 \times 224 \times 3$ images as inputs; the images are thus resized accordingly. As the images of our dataset are either binary or grey-scale, each image is replicated three times to obtain the desired form. The training and validation set is composed by $80 \%$ of the images of each dataset (more precisely, $64 \%$ of the dataset is used for training and $16 \%$ for validation), while the testing set is composed by the remaining $20 \%$ of the images.

The CNN classifier was tested multiple times with different input parameters, in order to determine their suitable values for our data. The following parameters are set for each run: number of epochs (number of complete passes through the training dataset) $=15$, batch size (number of training samples that are fed to the classifier at once to reduce the computational time $)=40$ images, validation frequency (number of iterations after which the validation set is fed to classifier to test its accuracy and update the weights of the fully connected layers) $=20$ iterations, and learning rate (step size) $=0.0001$. These parameter values are the same for both experiments described below, i.e. for segmented and grey-scale images.

\subsubsection{Segmented images}

The CNN classifier was first tested on segmented images, in order to take into account the fact that we are interested in pore alignments for CoT while random/spiral distribution are expected in SPT. The prediction accuracy results on 72 test images are shown in Table 1.

\subsubsection{Grey-scale images}

As the CNN classifier showed remarkable improvement in classification with respect to the SVM performances, the classifier is tested on the more challenging set 
of grey-scale images of Figure 7. The prediction accuracy results on 92 test images are shown in Table 1.

\subsection{Discussion}

We first want to point out that one of the challenges we faced during the training procedure was the small size of the dataset. Indeed, we had to work with a reduced number of available samples.

Considering the methods applied to segmented images, the CNN method appears more accurate than the SVM one. Indeed, the accuracy rates for SVM roughly hit less than $80 \%$, while they are higher than $90 \%$ for CNN for most of the tested situations (see Table 1). Despite the differences between the classification methods, it could be that the features captured by $\mathrm{CNN}$ on segmented images are richer than those extracted from the lines obtained by the Hough transform on pores.

From these results, it appears that the Deep Learning method is more competent than the SVM Machine Learning method in classifying the testing set, where it provided significantly more accurate results. Moreover, CNN automatically performs the feature detection / extraction steps with transfer learning, whence only the classification network needs to be trained. For SVM however, each image had to be pre-processed in order to extract the retained features. Finally, SVM is 5 times more computationally costly (in time) than the $\mathrm{CNN}$ one.

Ultimately, the CNN procedure gives slightly better classification results from grey-scale images than from segmented images. Yet, some information might be lost during by the segmentation / pore extraction procedure.

\section{Conclusion}

Promising results were obtained with Machine Learning and Deep Learning algorithms for classification of ancient pottery samples, running on 2-D cross-sections extracted from a set of $\mu$-CT images. This will be useful to classify archaeological sherds which are often damaged and difficult to distinguish from noisy images. As CNN methods give good results, different configurations of these techniques can be further investigated. To improve the classification, we also plan to acquire more samples and images, with other modalities and at different resolutions.

However, one of the most important drawbacks of CNNs is their lack of interpretation in terms of modelling. The advantage of the more traditional image analysis approaches (like the SVM one) is that it provides archaeologists with visual and quantitative elements for image interpretation (e.g. the quantity of parallel pore lines in CoT sherds) that are very useful for their analysis of the manufacturing process. Many additional features can also be considered, as for example the positions of lines intersection points.

With the same goal, the Hough transform can be adapted in order to catch circular or spiral-like behaviour of pores in SPT images, see (Coli et al., 2021). Finally, note that higher dimensional versions of the Hough transform are able to detect surfaces in 3-D images, see (Beltrametti et al., 2013; Ballard, 1981). They could provide good results on sherd images.

The quantitative analysis proposed here to discriminate between CoT and SPT manufacturing techniques using pore distribution in $\mu$-CT scans of sherds can be applied to other pottery building processes.

These promising results address similar issues concerning other markers of the micro-structures inherited from the building process. More specifically, the spatial organisation of mineral inclusions also reflects the technical gestures implemented 
during pottery making. The present approach may provide useful complementary classification results on such inclusions, though their segmentation from $\mu$-CT images is more difficult than those of the pores due to the variety of materials (hence of intensities in images). Next step will be to jointly process these different markers in order to refine the diagnostic and to furnish more robust and accurate information.

\section{Acknowledgements}

This work was supported by the French National Research Agency, with the reference number ANR-15-IDEX-0001, IDEX UCA ${ }^{\mathrm{JEDI}}$ "Multiscale Tomography: Imaging and Modelling Ancient Materials, Technical Traditions and Transfers TOMAT" project and through the 3IA Cte dAzur Investments, with the reference number ANR-19-P3IA-0002.

We thank the reviewers for their comments and suggestions.

\section{REFERENCES}

Abe, S. (2010). Support Vector Machines for Pattern Classification. Springer Publishing Company, Incorporated, 2nd edition.

Ballard, D. (1981). Generalizing the Hough transform to detect arbitrary shapes. Pattern Recognition, 13(2):111-22.

Beltrametti, M., Massone, A., and Piana, M. (2013). Hough Transform of Special Classes of Curves. SIAM Journal on Imaging Sciences, 6(1):391-412.

Coli, V. L., Gomart, L., Pisani, D., Cohen, S., Blanc-Féraud, L., Leblond, J., and Binder, D. (2021). Microcomputed tomography for discriminating between different forming techniques in ancient pottery: New segmentation method and pore distribution recognition. Archaeometry, pages 1-16.

de Rezende, E. R., Ruppert, G. C., Théohilo, A., Tokuda, E. K., and Carvalho, T. (2018). Exposing computer generated images by using deep convolutional neural networks. Signal Processing: Image Communication, 66:113 - 126.

Dia, K. (2020). Machine learning for imaging issues in archaeology. Master's thesis, Université Savoie Mont Blanc.

Gomart, L. (2010). Variabilité technique des vases du Rubané récent du Bassin parisien (RRBP) et du Villeneuve-Saint-Germain (VSG) : un cas d'étude dans la vallée de l'Aisne. Bulletin de la Société préhistorique française, 107(3):537-48.

Gomart, L. (2014). Traditions techniques et production céramique au Néolithique ancien. Étude de huit sites rubanés du Nord-Est de la France et de Belgique. Leiden: Sidestone.

Gomart, L., Weiner, A., Gabriele, M., Durrenmath, G., Sorin, S., Angeli, L., Colombo, M., Fabbri, C., Maggi, R., Panelli, C., Pisani, D., Radi, G., Tozzi, C., and Binder, D. (2017). Spiralled patchwork in pottery manufacture and the introduction of farming to Southern Europe. Antiquity, 91(360):1501-14.

Krizhevsky, A., Sutskever, I., and Hinton, G. E. (2012). Imagenet classification with deep convolutional neural networks. In Proceedings of the 25th International Conference on Neural Information Processing Systems - Volume 1, NIPS'12, page 10971105, Red Hook, NY, USA. Curran Associates Inc.

Mukhopadhyay, P. and Chaudhuri, B. (2015). A survey of Hough Transform. Pattern Recognition, 48(3):993-1010. 
Salanova, L., Vieugué, J., and Gomart, L. (2010). Methods of study of large ceramic complexes: series and sets of Neolithic pottery from Kovachevo (Bulgaria). Arheologia Sofia, (3-4):7-23.

Szegedy, C., Wei Liu, Yangqing Jia, Sermanet, P., Reed, S., Anguelov, D., Erhan, D., Vanhoucke, V., and Rabinovich, A. (2015). Going deeper with convolutions. In 2015 IEEE Conference on Computer Vision and Pattern Recognition $(C V P R)$, pages 1-9.

Widodo, A. and Yang, B.-S. (2007). Support vector machine in machine condition monitoring and fault diagnosis. Mechanical Systems and Signal Processing, 21(6):2560 - 2574. 